\title{
Joint Evaluation Method for Soil Compaction Degree Based on Electrical Resistivity And Elastic Wave Property
}

\author{
Jie Song \\ Geotechnical and Structural Engineering Research Center, Shandong University, Jinan 250061, \\ China. \\ tom1442@sina.com
}

Keywords: Electrical resistivity, elastic wave velocity, soil compaction degree, regression analysis, joint evaluation

\begin{abstract}
The regulation of earth resistivity and elastic wave velocity with the change of compaction degree under different moisture contents is studied by experiment. In addition, regression analysis is conducted on the relationship between compaction degree and soil resistivity and elastic wave velocity. Based on this quantitative research, the method for joint evaluation of soil compaction degree with electrical resistivity and elastic wave velocity is proposed. This method breaks the narrow application range in which it is only suitable for one kind or category of soil. With electrical resistivity in joint evaluation method, survey line can be arranged in a large scale; the design requirement on compaction degree and its evenness in the whole area can be evaluated; the dissatisfaction about compaction degree in the area can be obtained. All of these cannot be realized by other methods. Therefore, this work takes this method as the general survey method in common area. With elastic wave velocity in joint evaluation method, compaction degree at specific spot can be surveyed as by traditional methods; compaction degree in specific depth can be measured. Therefore, this work takes this method as the method for secondary evaluation or evaluation of specific area after evaluation by spot test and resistivity method. Moreover, two methods in joint evaluation method can be verified by each other to ensure the correctness of measurement and make effective evaluation for soil compaction degree.
\end{abstract}

\section{Introduction}

In various projects of roadbed, subgrade, etc., the key factor to affect the engineering safety is the quality of soil compaction. Therefore, it is greatly significant to develop the research on evaluation method of soil compaction degree. However, the present method generally used for detection of soil compaction is too original and obsolescent to satisfy with the demands of various projects in rapid development[1]. Relevant scholars have explored some new methods for the detection of compaction degree for various projects in rapid development, such as roadbed and subgrade. These methods include nuclear moisture-density meter method[2,3] and detection method by Rayleigh wave in recent years[4,5]. However, with nuclear moisture-density meter method, the radioactive substance is harmful to human body. Therefore, other instruments for punching are required, while the measurement correctness will be affected. Moreover, the detection method by Rayleigh wave can only make qualitative analysis evaluation for compaction quality at present, but cannot make quantitative research on the relationship between velocity and compaction degree.

\section{Test method and results}

The soil sample used in test is the clay with dominant component of natural arable soil. Before the test, the soil sample is put into the dryer for drying according to relevant regulation. Then, the sample is divided into six parts, and different contents of water are added into the sample to make soil sample with different moisture contents. Moreover, the moisture content in different parts of sample is accurately measured. 
Test of electrical resistivity and elastic wave velocity is conducted for soil samples with different compaction degree under moisture contents of $5.9 \%, 8.6 \%, 9.5 \%$ and $12.8 \%$, respectively. The compaction degree used in this test is measured by cutting-ring method. Fig. 1 shows the curve of test results and fitting curve. According to above curves and relevant analysis, the regulations of electrical resistivity and elastic wave velocity with the change of compaction degree are as follows: (1) the electrical resistivity of soil will rapidly decrease with the increase of compaction degree; (2) the wave velocity of soil shows linear increase with the increase of compaction degree.

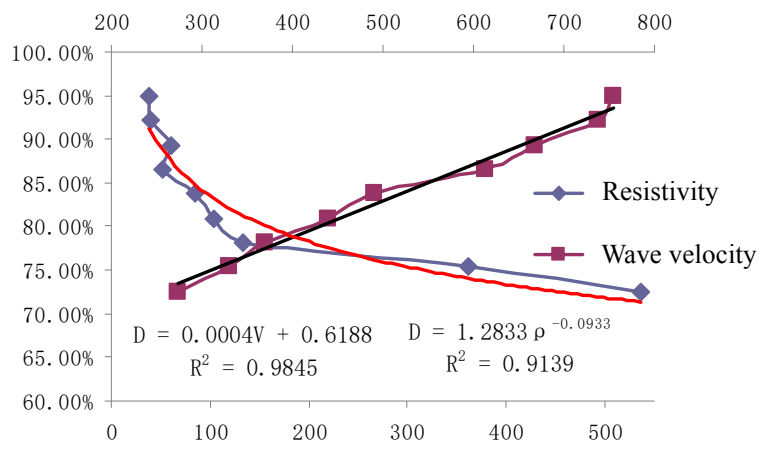

(a) the moisture content is $5.9 \%$

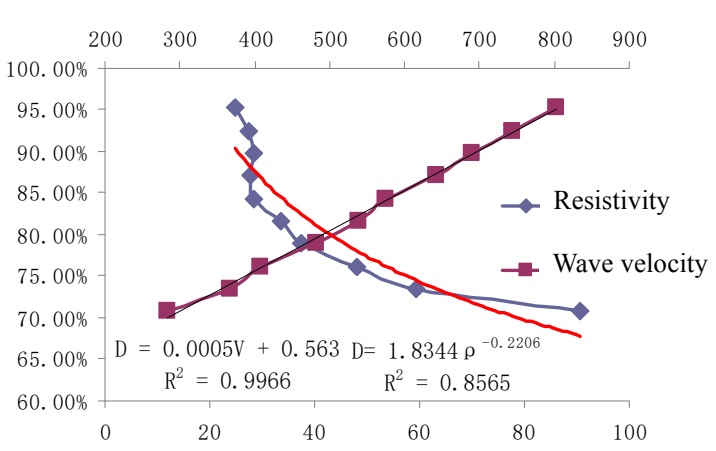

(b) the moisture content is $8.6 \%$

（b）含水率 $8.6 \%$ 时，压实度与电阻率、波速关系

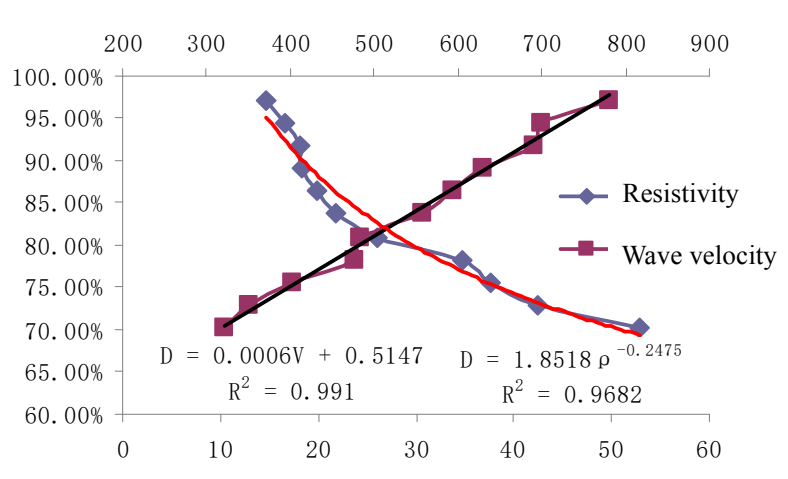

(c) the moisture content is $9.5 \%$

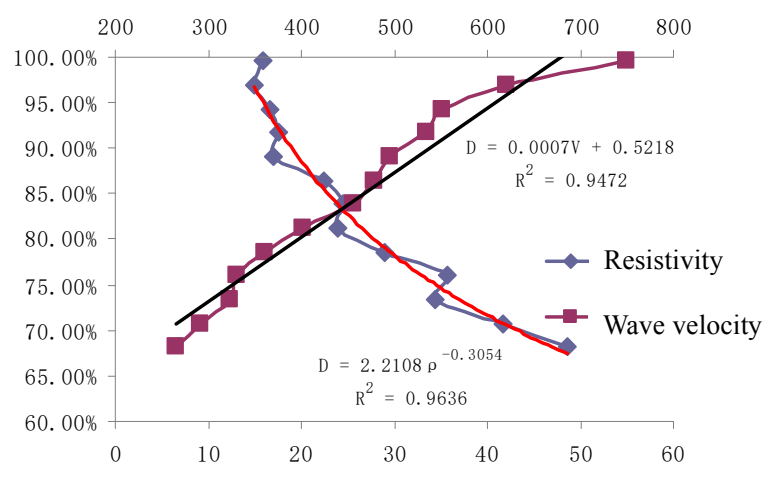

(d) the moisture content is $12.8 \%$

Fig. 1 The relationship between compaction degree and electrical resistivity and wave velocity

\subsection{Quantitative analysis on test results of the relationship between electrical resistivity and compaction degree}

By fitting the relationship between electrical resistivity and compaction degree in above figure, it shows that the electrical resistivity has correlation of power exponent with compaction degree. The fundamental formula of fitting is as follows:

$$
D=a r^{-b}
$$

In the formula, $D$ refers to soil compaction degree, $\rho_{\text {to electrical resistivity of soil, } a \text { and } b \text { to }}$ correlation coefficients.

In the formulas of relationship between soil compaction degree and electrical resistivity with different moisture contents, the values of $\mathrm{a}$ and $\mathrm{b}$ are different. Table 1 shows the specific relationship.

Table 1 Three Scheme comparing

\begin{tabular}{ccc}
\hline The moisture content & The fitting formula & $\mathrm{R}^{2}$ \\
\hline $5.9 \%$ & $D=1.2833 \rho^{-0.0933}$ & 0.9139 \\
$8.6 \%$ & $D=1.8344 \rho^{-0.2206}$ & 0.8565 \\
$9.5 \%$ & $D=1.8518 \rho^{-0.2475}$ & 0.9682 \\
$12.8 \%$ & $D=2.2108 \rho^{-0.9636}$ & 0.9636 \\
\hline
\end{tabular}


The difference of coefficient $a$ reflects the difference of moisture content in soil, while the difference of coefficient $b$ reflects the intensity of change of soil compaction degree with electrical resistivity. The larger the value of $b$ is, the more acute the change of electrical resistivity with moisture content will be. According to Table 1, the relevant coefficient is the close to 1 , so the fitting relationship is very good.

\subsection{Quantitative analysis on test results of the relationship between elastic wave velocity and compaction degree}

By fitting the relationship between elastic wave velocity and compaction degree in above figure, it shows that the relationship between wave velocity and compaction degree is approximately linear. The fundamental formula of fitting is as follows:

$$
D=c V+d
$$

In the formula, $D$ refers to soil compaction degree; $V$ to elastic wave velocity in soil; $c$ and $d$ to correlation coefficients.

In the formulas of relationship between soil compaction degree and elastic velocity with different moisture contents, the values of $c$ and $d$ are different. Table 2 shows the specific relationship.

Table 2 Three Scheme comparing

\begin{tabular}{ccc}
\hline The moisture content & The fitting formula & $\mathrm{R}^{2}$ \\
\hline $5.9 \%$ & $D=0.0004 V+0.6188$ & 0.9845 \\
$8.6 \%$ & $D=0.0005 V+0.563$ & 0.9966 \\
$9.5 \%$ & $D=0.0006 V+0.5147$ & 0.991 \\
$12.8 \%$ & $D=0.0007 V+0.5218$ & 0.9474 \\
\hline
\end{tabular}

The difference of coefficient $c$ reflects the intensity of change of soil compaction degree with electrical resistivity. The larger the value of $c$ is, the more acute the change of electrical resistivity with moisture content will be. The difference of coefficient $d$ reflects the influence of different moisture contents. The value of $d$ will decrease with the increase of moisture content.

\section{Joint evaluation of electrical resistivity and elastic wave velocity for soil compaction degree}

The method of joint evaluation by electrical resistivity and elastic wave velocity for soil compaction degree is proposed based on above research.

With electrical resistivity in joint evaluation method, measuring line can be arranged in a large scale. Resistivity distribution in the whole area can be obtained by arranging several measuring lines in high density. Then, the distribution of compaction degree can be obtained according to the quantitative relation between electrical resistivity and compaction degree of soil. With this method, compaction condition in the whole area can be seen in the figure of electrical results, and the compaction can be evaluated if it is satisfied with design and uniform. Moreover, area dissatisfying with compaction degree in area can be found, which cannot be realized by other methods. Therefore, this work takes electrical resistivity method as general survey method in conventional area.

Elastic wave velocity in joint evaluation method is taken as the method for secondary evaluation and evaluation for special area after spot test and electrical resistivity method. As traditional method, elastic wave velocity can test the compaction degree at any specific spot. Moreover, compaction degree at any specific depth can be measured with this method.

The secondary evaluation refers to the secondary measurement for some abnormal areas (areas with compaction degree higher or lower the requirement) with elastic wave velocity after obtaining the electrical resistivity of the whole area by electrical resistivity method. The measurement in specific area refers to areas in which survey lines cannot be arranged or near which special conditions, including interference of low resistivity exist. 


\section{Summary}

Firstly, the relationship between compaction degree and electrical resistivity and elastic wave velocity under certain moisture contents is discussed in this paper. Based on the conclusion, analysis and fitting of test data, the empirical formulas for relationship between compaction degree and electrical resistivity and elastic wave velocity is proposed, and the relationship between the coefficient in empirical formula and moisture content is analyzed. Finally, the relationship between compaction degree and electrical resistivity and elastic wave velocity is concluded. Based on the relationship, he joint evaluation method with electrical resistivity and elastic wave velocity for soil compaction degree is proposed.

\section{References}

[1] SHA Qing-lin. Road Compaction and Compaction St andard[M]. Beijing : China Communications Press, 1999.

[2] WANG Chao-dong, XU Xi-chang, GUO Jian-yang. Application of Nuclear Measuring Technique in Geotechnical Engineering[J]. Chinese Journal of Rock Mechanics and Engineering,2001, 20 ( S1 ) : 1900-1902.

[3] Chi Xi-gao. Application of Nuclear Densimeter in Layered Compaction of a Filling Project[J]. Soil Eng. and Foundation, 1997,04:52-53.

[4] LIU Jiang-ping, LUO Yin-he, HE Wei-bing. Method of neighboring trace transient Rayleigh wave and its application in compactness inspection[J]. Chinese Journal of Geotechnical Engineering,2009, 11:1652-1659.

[5] CHAI Hua- you, WANG Jiang-bo, ZHOU Yi-qin, et al. Progress of Rayleigh Wave Analysis Method and Its Application[J]. Chinese Journal of Rock Mechanics and Engineering, 2002, 21(1): 119125. 